Dekker, J., Dijk, G.M. van, Veenhof, C. Risk factors for functional decline in osteoarthritis of the hip or knee. Current Opinion in the Rheumatology: 2009, 21(5), 520-524

\begin{tabular}{|l|l|}
\hline $\begin{array}{l}\text { Postprint } \\
\text { Version } \\
\text { Journal website }\end{array}$ & 1.0 \\
$\begin{array}{l}\text { http://journals.lww.com/co-rheumatology/Abstract/publishahead/Risk factors } \\
\text { for functional decline in.99945.aspx }\end{array}$ \\
\hline Pubmed link & $\underline{\text { http://www.ncbi.nlm.nih.gov/pubmed/19550331 }}$ \\
\hline DOI & $10.1097 /$ BOR.0b013e32832e6eaa \\
\hline
\end{tabular}

This is a NIVEL certified Post Print, more info at http://www.nivel.eu

\title{
Risk factors for functional decline in osteoarthritis of the hip or knee
}

\author{
JOOST DEKKER ${ }^{A, B}$, GABRIELLA M. VAN DIJK ${ }^{C}$ AND CINDY VEENHOF ${ }^{C}$
}

${ }^{\mathrm{a}}$ Department of Rehabilitation Medicine,

bepartment of Psychiatry, EMGO Institute, VU University Medical Centre, Amsterdam and cNIVEL (Netherlands Institute for Health Services Research), Utrecht, The Netherlands

\begin{abstract}
Purpose of review: To summarize recent studies on risk factors for functional decline (i.e. worsening of pain and activity limitations) in osteoarthritis of the hip or knee; after a long period of neglect, information on risk factors for functional decline has markedly improved in recent years. Recent findings: Studies show slow worsening of pain and activity limitations over time. There is a considerable between-individual variation in the course of pain and activity limitations; identification of risk factors for functional decline is therefore highly relevant. Risk factors for functional decline include characteristic physical impairments in osteoarthritis (pain, stiffness, reduced muscle strength, laxity of the knee joint, proprioceptive inaccuracy, poor standing balance and impaired range of joint motion), cognitive and visual impairments, comorbidity and overweight, psychological and social factors (anxiety, depression, fatigue, poor self-efficacy and social support), health behaviors and sociodemographic factors. Summary: The information on risk factors can be used pragmatically to construct prediction rules on functional decline in osteoarthritis patients. Both clinicians and patients value the ability to prognosticate future pain and activity limitations. Furthermore, the information on risk factors can be used to construct causative models to explain pain and activity limitations: examples include 'instability of joints' and 'avoidance of activity' as causal mechanism of pain and activity limitations. Improved understanding of pain and activity limitations is the basis for improved treatment and rehabilitation of osteoarthritis patients.
\end{abstract}

\section{INTRODUCTION}

Pain and activity limitations such as problems in walking, rising up, sitting down and stair climbing are primary symptoms of osteoarthritis of the hip or knee. Advancing knowledge of whether pain and activity limitations improve or deteriorate over time, and which factors predict functional decline, is of benefit to patients, clinicians and scientists. For a long time, 
Dekker, J., Dijk, G.M. van, Veenhof, C. Risk factors for functional decline in osteoarthritis of the hip or knee. Current Opinion in the Rheumatology: 2009, 21(5), 520-524

risk factors for functional decline (i.e. worsening of pain and activity limitations) have been surprisingly little studied. In our previous review covering the literature until March 2005 [1], we identified a limited number of high-quality studies; in fact, only one high-quality prognostic study [2] was available. At present, we will review recent prognostic studies on risk factors for functional decline (i.e. worsening of pain and activity limitations) in osteoarthritis of the hip or knee, taking our previous review as a starting point.

\section{COURSE OF PAIN AND ACTIVITY LIMITATIONS}

Our previous review [1] showed that pain and activity limitations in hip and knee osteoarthritis are slowly deteriorating, with evidence for worsening after 3 years of followup. These results are confirmed by recent studies. Dunlop et al. [3] reported functional decline in $13.6 \%$ of patients with arthritis over a period of 2 years. Mallen et al. [4] reported poor functional outcome in $60 \%$ at 18 months of follow-up in older community-dwelling adults with knee pain ('poor outcome' also included patients whose status continued to be poor, that is, poor at baseline as well as follow-up). van Dijk et al. [5] found that performance-based activity limitations remained unchanged after a follow-up of 3 years in patients with confirmed osteoarthritis of the hip or knee; these patients showed a slight, not clinically relevant, improvement in self-reported activity limitations. Peters et al. [6] found that pain and disability generally deteriorated over 7 years in a community-based sample of adults with hip and knee pain; however, quite a number of individuals (about 30\%) improved. In meniscectomized patients at risk for osteoarthritis, knee-related pain and physical function worsened over 7 years [7]. Covinsky et al. [8] demonstrated that functional decline is worse in patients with arthritis than in those without arthritis; patients with arthritis were substantially more likely to develop persistent activity limitations over 10 years of follow-up than those without arthritis. This study is exceptional in comparing patients with arthritis with a control group without arthritis, thereby clearly demonstrating the specific impact of arthritis on functional decline. Overall, these data show slow worsening of pain and activity limitations over time. The data also point to a considerable between-individual variation in the course of pain and activity limitations; some patients seem to be stable or to even improve, whereas others deteriorate. Identification of risk factors for functional decline is, therefore, highly relevant.

\section{RISK FACTORS}

Risk factors for functional decline in osteoarthritis are categorized as physical impairments, cognitive and visual impairments, comorbidity and bodyweight, psychological and social factors, health behaviors and sociodemographic factors.

\section{Physical impairments}

Osteoarthritis is associated with characteristic physical impairments. In our previous review [1], we found evidence that these physical impairments predict future functional decline. Several recent studies have elaborated on the prognostic value of physical impairments. In older community-dwelling adults with knee pain, Mallen et al. [4] found pain at baseline to predict poor functional outcome at 18 months of follow-up. Worsening of pain has been found to have prognostic value as well, in addition to the prognostic value of pain at baseline: van Dijk et al. [5 ] found that increased pain at 1-year followup predicts activity limitations at 3 years of follow-up in patients with established osteoarthritis of the hip or knee. This finding suggests accelerated functional decline in a subgroup of patients showing increased pain at 1-year follow-up. Thomas et al. [9 ] reported that the number of tender points at baseline and stiffness at baseline predict poor functional outcome at 18 months of follow-up. Similarly, in meniscectomized patients at high risk for osteoarthritis, a symptomatic knee predicted more pain and poorer functional outcome at 2 years of followup [10]. In our previous review, we found evidence that reduced muscle strength, laxity of 
Dekker, J., Dijk, G.M. van, Veenhof, C. Risk factors for functional decline in osteoarthritis of the hip or knee. Current Opinion in the Rheumatology: 2009, 21(5), 520-524

the knee joint and proprioceptive inaccuracy predict functional decline. These findings have been confirmed recently: greater muscle strength predicts less pain and better physical functioning at 30months of follow-up in knee osteoarthritis [11]. Furthermore, reduction of muscle strength at 1-year follow-up predicts activity limitations at 3 years of follow-up in knee osteoarthritis [5 ]. Single-leg standing balance at baseline predicts poor functional outcome at 18months of follow-up [9 ]. Traditionally, reduction of muscle strength has been regarded as the major cause of activity limitations, especially in knee osteoarthritis. Recent evidence points out that, in addition to muscle weakness, instability of the knee joint affects activity limitation as well. Instability of the knee (i.e. inability of the knee to maintain a position or to control movements under different external loads) contributes greatly to activity limitations [12] and is affected by muscle weakness, laxity of the joint, poor proprioception and varus-valgus movement in the knee joint (movement in the knee in the frontal plane) [13,14,15 ]. Thus, muscle weakness has a direct effect in limiting activity, but muscle weakness also interacts with other factors - laxity, poor proprioception and varusvalgus movement - resulting in instability of the knee joint: this also limits activity (see Fig. 1). The impact of instability on activity limitation has so far been evaluated in cross-sectional studies. The prognostic value of instability of the knee joint is still to be determined. A new finding is that reduction of range of joint motion (ROM) at 1-year follow-up predicts activity limitations at 3 years of follow-up [5 ]. Impaired ROM is a characteristic feature of osteoarthritis. Although impaired ROM has a strong impact on functioning [16], ROM is not frequently studied, and the importance of impaired ROM for functioning seems to be somewhat underestimated. The recent finding that reduction of ROM at 1-year follow-up predicts activity limitations may help to focus research efforts on ROM. Because of the strong impact on functioning, impairments of ROM in osteoarthritis of the hip and knee certainly need to be studied. With regard to radiological signs of osteoarthritis and future functional outcome, both positive [9] and negative [10] findings have been reported, confirming previous conflicting reports [1].

\section{[FIGURE 1]}

\section{Cognitive and visual impairments}

Prevalence of osteoarthritis is strongly associated with age: most osteoarthritis patients are older people. Ageing frequently brings impairments, including cognitive and visual impairments. Recently, cognitive and visual impairments at baseline were found to predict functional decline over a 2-year period in patients with arthritis [3]. Similarly, cognitive functioning and ear-eye-nose-throat disease (primary visual impairment) predicted functional decline in hip osteoarthritis [5 ]. These findings highlight the functional significance of impairments associated with old age, despite the fact that these impairments are not intrinsically associated with osteoarthritis. From the perspective of rehabilitation of functioning in osteoarthritis, these findings are highly relevant.

\section{Comorbidity and bodyweight}

Recent studies found that comorbidity is an important risk factor for functional decline. Higher morbidity count predicted worsening of activity limitations in patients with confirmed osteoarthritis of the hip or knee [5 ]. In a community-based sample of adults with hip and knee pain, cardiovascular morbidity was found to be a risk factor forworsening of pain and disability in hip osteoarthritis and hypertension for knee osteoarthritis [6].

Likewise, van Dijk et al. [5 ] found cardiac disease to predict worsening of self-reported and performance-based activity limitations in patients with confirmed hip osteoarthritis. Diabetes and stroke were also found to be predictors of functional decline in patients with arthritis [3]. Finally, evidence was found that poorer general health was associated with poor functional outcome after 18 months [4]. Similarly, 'other health problems' were associated with worsening of pain and disability for both hip and knee osteoarthritis [6]. Covinsky et al. [8 ] 
Dekker, J., Dijk, G.M. van, Veenhof, C. Risk factors for functional decline in osteoarthritis of the hip or knee. Current Opinion in the Rheumatology: 2009, 21(5), 520-524

demonstrated that patients with both arthritis and another chronic condition were at greater nive risk for developing activity limitations compared with patients with arthritis only. Comorbidity increases the likelihood of functional decline; the combined influence of comorbidity and osteoarthritis is greater than would be expected from the influence of either comorbidity or osteoarthritis alone [17]. Comorbidity brings physiological, sensorimotor and psychological impairments: examples include limited exercise tolerance in chronic heart failure, muscle atrophy in chronic obstructive pulmonary disease (COPD) and avoidance of exercise out of fear for recurrent myocardial infarction, respectively. These impairments probably explain the additional impact of comorbidity on functional decline. The precise mechanisms of the influence of comorbidity on functional decline in osteoarthritis remain to be elucidated, however. Recent studies confirm the previous finding [1] on bodyweight as a risk factor for future limitations in activities in knee osteoarthritis. In older communitydwelling adults with knee pain, overweight and obesity were prognostic indicators of poor functional outcome after 18 months [4]. Likewise, a higher BMI predicted worsening of pain and disability over 7 years for knee osteoarthritis [6]. In meniscectomized patients at risk for knee osteoarthritis, both overweight and obesity were risk factors for clinically relevant change in physical function over 2 years but not for pain [10]. Body weight influenced change in performance over 7 years but not in self-reported limitations and pain [7]. We have not been able to confirm these findings, possibly because of a high number of potential predictors already in the analysis [5].

\section{Psychological and social factors}

Good mental health, better self-efficacy and social support have been previously identified as protective factors for functional decline in patients with knee osteoarthritis; in the absence of high-quality studies, we did not reach a conclusion on the impact of these factors in hip osteoarthritis [1]. Recent studies have elaborated on the role of psychological and social factors in functional decline. Mallen et al. [4] found that anxiety was one of the factors predicting poor functional outcome after 18 months in older patients with knee pain. Depression was associated with poor functional outcome in that study as well. This last finding has also been reported by Dunlop et al. [3], who concluded that depressive symptoms were a significant predictor of functional limitations of patients with arthritis over a 2-year period. Similarly, low vitality or fatigue (as measured with the SF-36) [18] has a negative impact on the course of activity limitations after 3 years of follow-up in patients with knee osteoarthritis (van Dijk GM, et al., in preparation). The impact of psychological factors on functional decline can be interpreted in terms of avoidance of activity $[19,20]$. Osteoarthritis patients tend to avoid activity, because activity induces pain. The avoidance of activity is a two edged sword, however. A low activity level induces muscle weakness, instability of joints and activity limitations. Negative affect (e.g. anxiety, depression and low vitality) is thought to strengthen the tendency to avoid activity, resulting in even more activity limitations. This mechanism explains the impact of negative affect on functional decline in osteoarthritis patients

\section{[FIGURE 2]}

\section{Health behaviors}

Previously, aerobic exercise was found to protect against functional decline [1]. Recently, it was found that lack of regular vigorous activity almost doubled the odds of functional decline in patients with arthritis [3]. Surprisingly, the use of alcohol was found to protect against functional decline [3].

\section{Sociodemographic factors}

In our review, older age was identified as a risk factor for functional decline in patients with knee osteoarthritis [1]. Several studies have elaborated on the prognostic value of age. Mallen et al. [4] found that older age was associated with poor functional outcome after 18 
Dekker, J., Dijk, G.M. van, Veenhof, C. Risk factors for functional decline in osteoarthritis of the hip or knee. Current Opinion in the Rheumatology: 2009, 21(5), 520-524

months in patients with knee pain. van Dijk et al. [5 ] identified older age as a risk factor for activity limitations after 3 years of follow up in patients with hip osteoarthritis. And finally, Roos et al. [7] found that older age negatively influenced objectively assessed physical function in patients at high risk for knee osteoarthritis or with knee osteoarthritis. These studies point to the impact of as yet unidentified age-related impairments on functional decline, as age in itself does not explain functional decline. Contrary to the previous results [1], an association between sex and functional decline was found in two studies: women showed more functional decline than men in both studies [3,7]. Another new finding is the more pronounced functional decline among certain ethnic groups (more decline in AfricanAmerican or Hispanic-Americans compared with Whites) [3]. Finally, lower social class and being retired were identified as risk factors for worsening of pain and disability in patients with osteoarthritis [6].

\section{[TABLE 1]}

\section{CONCLUSION}

We conclude this short review with the following remarks:

(1) Recent studies confirm that pain and activity limitations in osteoarthritis of the knee or hip are slowly deteriorating. The data also point to considerable variation between patients; some patients seem to be stable or to even improve, whereas others deteriorate. Identification of risk factors for functional decline is, therefore, highly relevant.

(2) Risk factors for functional decline have been summarized in Table 1. The table shows a range of risk factors, which all have been shown to predict more functional decline (i.e. worsening of pain and activity limitations) in prospective studies.

(3) The information on risk factors can be used pragmatically to construct prediction rules on functional decline in osteoarthritis patients; both clinicians and patients value the ability to prognosticate future pain and activity limitations. Furthermore, the information on risk factors can be used to construct causative models to explain future pain and activity limitations; improved understanding of pain and activity limitations is the basis for improved treatment and rehabilitation of osteoarthritis patients. Examples include the model on instability of joints (see Fig. 1); exercise therapy targeting instability is expected to result in improved outcome. Similarly, the model on avoidance of activity (see Fig. 2) can give guidance to the behavioral approach towards functional decline in osteoarthritis. Because of the impact of impaired ROM on functional decline, explanatory models and treatment should target ROM as well.

(4) Osteoarthritis synergizes with coexisting conditions to contribute to functional decline. The impact of comorbidity and overweight on functional decline has been convincingly demonstrated in recent studies. Furthermore, cognitive and visual impairments have an impact on functional decline. Future research should unravel how coexisting disorders (i.e. comorbidity, overweight and cognitive and visual impairments) have an impact on functional decline in osteoarthritis.

(5) In our review, we included studies on patients with a diagnosis of hip or knee osteoarthritis as well as studies on older patients with knee pain and patients with arthritis: osteoarthritis is the most likely diagnosis in the latter patients. We excluded studies on patients who clearly had a mix of diagnoses [21]. Obviously, better standardization of the diagnosis of osteoarthritis is desirable. Other methodological issues include the lack of standardization of terminology on functional decline, the lack of consensus on the assessment of outcome, as well as differences between studies in the nature and number of covariates in the analyses.

(6) In conclusion, despite these methodological issues, knowledge on risk factors for functional decline in osteoarthritis of the hip or knee seems to be rapidly proliferating. 
Dekker, J., Dijk, G.M. van, Veenhof, C. Risk factors for functional decline in osteoarthritis of the hip or knee. Current Opinion in the Rheumatology: 2009, 21(5), 520-524

\section{ACKNOWLEDGEMENT}

The writing of this manuscript was in part supported by a grant from the Netherlands Organization for Health Research and Development (ZON-MW Revalidatieonderzoek).

\section{REFERENCES AND RECOMMENDED READING}

Papers of particular interest, published within the annual period of review, have been highlighted as: of special interest of outstanding interest Additional references related to this topic can also be found in the Current World Literature section in this issue (pp. 000000).

1 van Dijk GM, Dekker J, Veenhof $\mathrm{C}$, van den Ende $\mathrm{CH}$. Course of functional status and pain in osteoarthritis of the hip or knee: a systematic review of the literature. Arthritis Rheum 2006; 55:779-785.

2 Sharma L, Cahue S, Song J, et al. Physical functioning over three years in knee osteoarthritis: role of psychosocail, local mechanical and neuromuscular factors. Arthritis Rheum 2003; 48:3359-3370.

3 Dunlop DD, Semanik P, Song J, et al. Risk factors for functional decline in older adults with arthritis. Arthritis Rheum 2005; 52:1274-1282.

4 Mallen CD, Peat G, Thomas E, et al. Predicting poor functional outcome in communitydwelling older adults with knee pain: prognostic value of generic indicators. Ann Rheum Dis 2007; 66:1456-1461.

5 van Dijk GM, Veenhof C, Coene N, et al. Course of limitations in activities in osteoarthritis of the hip or knee: the influence of body functions, comorbidity and cognitive functioning. Arch Phys Med Rehabil (accepted subject to minor revision). This study evaluates the impact of physical and cognitive impairments and comorbidity on functional decline. The study emphasizes the role of comorbidity and physical impairments, including ROM and other physical impairments.

6 Peters TJ, SandersC, Dieppe P, Donovan J. Factors associatedwith change in pain and disability over time: a community-based prospective observational study of hip and knee osteoarthritis. Br J Gen Pract 2005; 55:205- 211.

7 Roos EM, Bremander AB, Englund M, Lohmander LS. Change in self-reported outcomes and objective physical function over 7 years in middle-aged subjects with or at high risk of knee osteoarthritis. Ann Rheum Dis 2008; 67:505-510.

8 Covinsky KE, Lindquist K, Dunlop DD, et al. Effect of arthritis in middle age on older-age functioning. J Am Geriatr Soc 2008; 56:23-28. This study desribes functional decline over 10 years of follow-up. This study is exceptional in comparing patients with arthritis with a control group without arthritis, thereby clearly demonstrating the specific impact of arthritis on functional decline.

9 Thomas E, Peat G, Mallen C, et al. Predicting the course of functional limitation among older adults with knee pain: do local signs, symptoms and radiographs add anything to general indicators? Ann Rheum Dis 2008; 67:1390-1398. This study explores the additional prognostic value of clinical history, physical examination and radiographic findings to a previously derived simple generic model (age, BMI, anxiety and pain severity). Although of limited value for clinical purposes, the study did identify several additional prognostic factors

10 Paradowski PT, Englund M, Lohmander LS, Roos EM. The effect of patient characteristics on variability in pain and function over two years in early knee osteoarthritis. Health Qual Life Outcomes 2005; 3:59.

11 Amin S, Baker K, Niu J, et al. Quadriceps strength and the risk of cartilage loss and symptom progression in knee osteoarthritis. Arthritis Rheum 2009; 60:189-198.

12 Felson DT, Niu J, McClennan C, et al. Knee buckling: prevalence, risk factors, and associated limitations in function. Ann Intern Med 2007; 147:534- 540.

13 van der Esch M, Steultjens M, Knol DL, et al. Joint laxity and the relationship between muscle strength and functional ability in patients with osteoarthritis of the knee. Arthritis Rheum 2006; 55:953-959.

14 van der Esch M, Steultjens M, Harlaar J, et al. Joint proprioception, muscle strength, and functional ability in patients with osteoarthritis of the knee. Arthritis Rheum 2007; 57:787793. 
Dekker, J., Dijk, G.M. van, Veenhof, C. Risk factors for functional decline in osteoarthritis of the hip or knee. Current Opinion in the Rheumatology: 2009, 21(5), 520-524

15 van der Esch M, Steultjens M, Harlaar J, et al. Varus-valgus motion and functional ability i in patients with knee osteoarthritis. Ann Rheum Dis 2008; 67:471-477. This study addresses the impact of objectively assessed varus-valgus motion of the knee on functional ability. Results are interpreted in terms of the instability of the knee.

16 Steultjens MP, Dekker J, van Baar ME, et al. Range of joint motion and disability in patients with osteoarthritis of the knee or hip. Rheumatology (Oxford) 2000; 39:955-961. 17 Kadam UT, Croft PR. Clinical comorbidity in osteoarthritis: associations with physical function in older patients in family practice. J Rheumatol 2007; 34:1899-1904.

18 Aaronson N, Muller M, Cohen P, et al. Translation, validation, and norming of the Dutch language version of the SF-36 Health Survey in community and chronic diseases. J Clin Epidemiol 1998; 51:1055-1068.

19 Dekker J, Tola P, Aufdemkampe G, Winckers M. Negative affect, pain and disability in osteoarthritis patients: the mediating role of muscle weakness. Behav Res Ther 1993; 31:203-206.

20 Steultjens MP, Dekker J, Bijlsma JW. Avoidance of activity and disability in patients with osteoarthritis of the knee: the mediating role of muscle strength. Arthritis Rheum 2002; 46:1784-1788.

21 Belo JN, Berger MY, Koes BW, Bierma-Zeinstra SM. Prognostic factors in adults with knee pain in general practice. Arthritis Rheum 2009; 61:143-

FIGURES AND TABLES

Figure 1 Instability of the knee and activity limitations

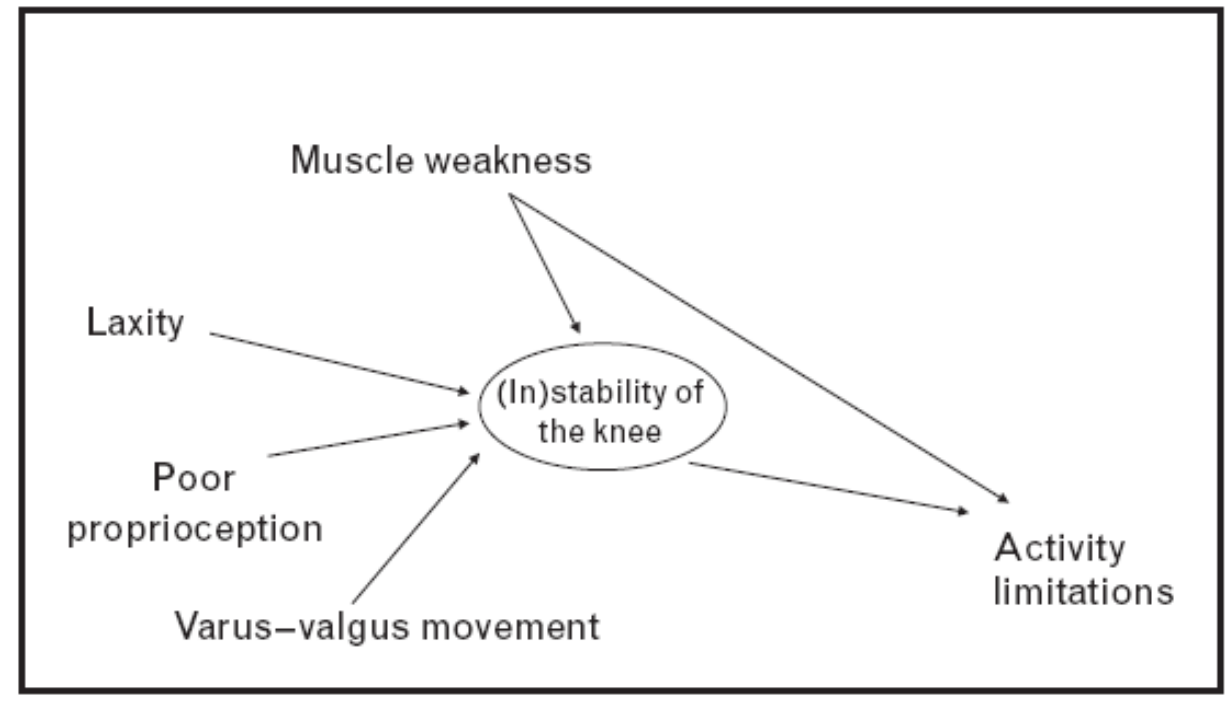

Stability: ability of the knee joint to maintain a position or to control movements under differing external loads. 
Figure 2 Avoidance of activity and activity limitations

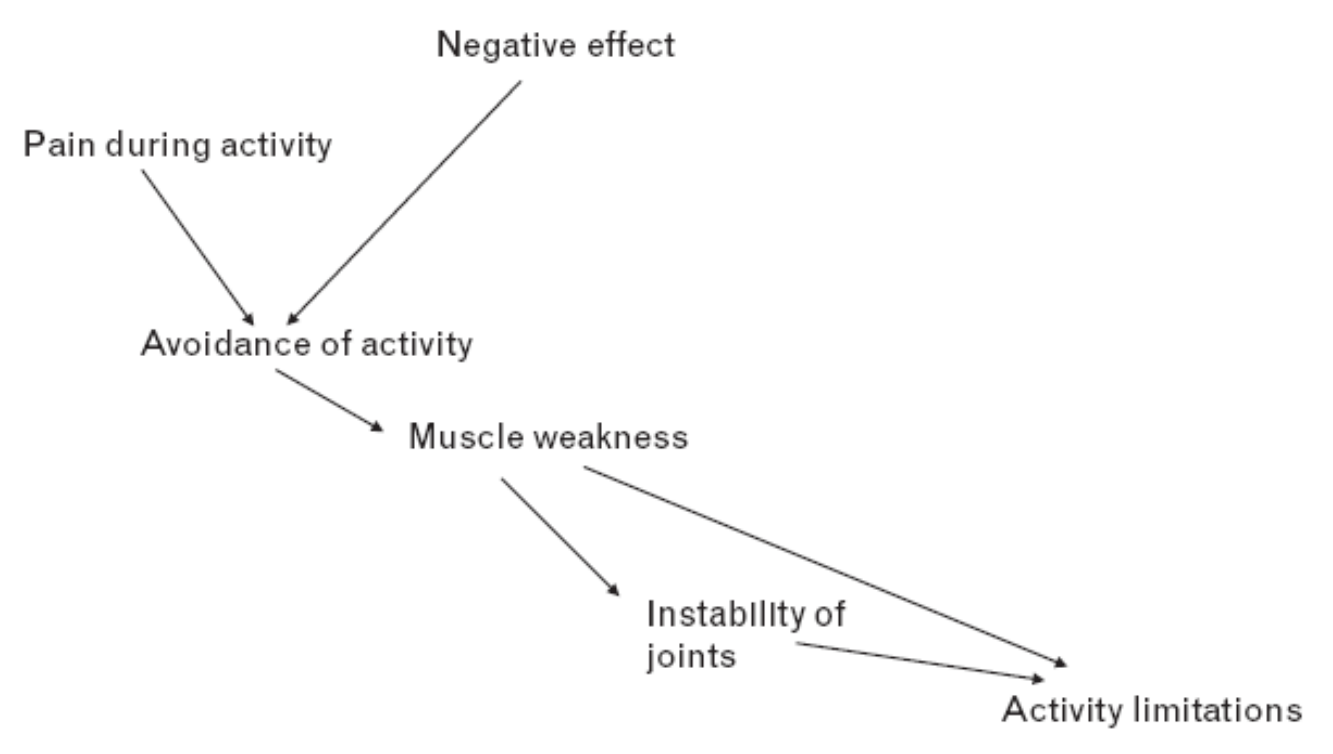

Table 1 Risk factors for functional decline in osteoarthritis of the hip or knee

Physical impairments

Cognitive and visual impairments

Comorbidity and overweight

Psychological and social factors

Health behaviors

Sociodemographic factors

Pain, worsening of pain, stiffness and tender points

Reduced muscle strength, deterioration of muscle strength, laxity of the knee joint, proprioceptive inaccuracy and standing balance

Deterioration of range of joint motion

Morbidity count and specific diagnoses

Poor mental health, self-efficacy and social support; anxiety, depression, low vitality and fatigue

Lack of vigorous activity Higher age, female sex, ethnicity, lower social class and being retired 